\section{MEDICO RESEARCH CHRONICLES ISSN NO. 2394-3971 \\ DOI No. 10.26838/MEDRECH.2021.8.6.570}

OPEN ACCESS

Contents available at www.medrech.com

\title{
PREMATURE RUPTURE OF MEMBRANE AND PERINATAL OUTCOME
}

Pratima Rani Biswas, Mamata Manjuri, Shamima Rahman, Osman Goni

Assistant Professor (Gynae \& Obs), Colonel Malek Medical College, Manikganj, Bangladesh

\section{ARTICLE INFO}

Article History

Received: October 2021

Accepted: November 2021

Keywords Premature rupture of membrane, chorioamnionitis, Prematurity.

\section{ABSTRACT}

Original ReSEarCh Article

Objective: To find out the complications of a pregnant woman with premature rupture of membrane (PROM) and assess the outcome of it. Materials and Methods: This prospective study was carried out at the Department of Obstetrics \& gynecology of Coronel Malek Medical College Hospital, Manikgonj, Bangladesh from January 2020 to December 2020. All pregnant women admitted with PROM during this period were taken as the study population. After taking proper history they were categorized as a term or preterm PROM on the basis of duration of gestation estimated from $1^{\text {st }}$ day of last menstrual period (LMP), previous antenatal records, clinical examination \& also previous ultrasonography (USG) reports. Out of sociodemographic characteristics, age distribution and gravidity were noted. The diagnosis was done on the basis of examination and investigations. All the women with PROM were then followed up, management was given according to protocol and a cesarean section was performed according to indication. Birth weight and Apgar score of newborns were noted after delivery. Results: There were 297 cases of PROM recorded among 4461 admitted obstetrical patients over one year period. The hospital incidence of PROM was found to be $6.6 \%$. Among them, $57.6 \%$ of patients were admitted at term and $42.4 \%$ of patients came before 37 completed weeks of gestation. Most of the pregnant women were between $20-24$ years of age (43.7\%), 43.2\% were primigravida $(56.6 \%)$ were multigravida. About $48.5 \%$ of women presented with different complications associated with PROM. Among which $15.7 \%$ patients had oligohydramnios, $8.5 \%$ of patients presented with chorioamnionitis suggested by culture report of the high vaginal swab. Urine for culture and sensitivity was also done. About five $(5.2 \%)$ developed premature labour before 37 th week of gestation. About $10 \%$ of women developed obstetric complications (failed trial) associated with medical diseases. Seventy-seven patients were delivered by caesarean section, $16.2 \%$ vaginally, $4.04 \%$ patients responded to conservative management and $2.5 \%$ patients were referred to tertiary centre for extreme prematurity with associated complicating factors. Most of the babies (38.4\%) were born with birth weight between 2.1- 
Corresponding author Pratima Rani Biswas *
$2.5 \mathrm{~kg}$ and $10.3 \%$ babies were less than $1.5 \mathrm{~kg}$. Conclusion: Term PROM was more in comparison to PPROM and most of them were multigravida. Cesarean section rate was high. Most common complication of was subclinical urogenital infection (53\%) next oligohydramnios $(25 \%) \&(13.5 \%)$ women presented with chorioamnionitis.

2021, www.medrech.com

\section{INTRODUCTION}

Premature rupture of membrane (PROM) is a syndrome characterized by spontaneous rupture of the chorioamnion before the onset of uterine contractions ${ }^{1}$. It occurs in approximately $10 \%$ of all pregnancies $^{2}$. The membranes may rupture either at term, that is after 37 completed gestational weeks, or before term, when it is called preterm prelabour rupture of membranes (PPROM). In these cases, there is serious concern regarding obstetric complications as well as perinatal morbidity and mortality ${ }^{1}$. In the majority of the condition causes is not known ${ }^{3}$. Theoretically, PROM may occur because of increased friability of the membranes, decreased tensile strength of the membrane or an increase in intrauterine pressure or both ${ }^{1-3}$. Under normal circumstances, the tensile strength of the membranes increases until 20 weeks and then plateaus until 39 weeks when it starts to decrease dramatically. The amniotic membranes are connective tissue structure and their tensile strength depends on the synthesis, degradation, and quality of their collagen ${ }^{2}$. An abnormal collagen structure may be responsible for PROM as evidenced by the high frequency of PROM in women affected by connective tissue disorders such as the Ehlers-Danlos syndromeu†. Also, tobacco smoking and nutritional deficiencies of zinc, copper, and ascorbic acid cause abnormal collagen crosslinking and may produce $\mathrm{PROM}^{2}$. intraamniotic infection may cause alterations in the tensile strength of the fetal membranes by mechanisms different than those involved in the increase in collagenolytic activity. Several of the microorganisms found in women with
PPROM and amniotic infection produce proteolytic enzymes that can weaken the fetal membranes. Furthermore, the host inflammatory response to intra-amniotic infection almost certainly plays a role in the mechanism of PPROM. This inflammatory response includes the production of cytokines that stimulate prostaglandin production by the amnion and chorion. Prostaglandins stimulate uterine contractility and cause increased collagen degradation. Also, the glucocorticoids produced in response to the stress of the intra-amniotic infection may be involved in facilitating rupture of the membranes ${ }^{2}$. PROM is a significant occurrence as it can cause maternal complications, increased operative procedures, and perinatal morbidity and mortality. The obstetrician is often in a dilemma regarding the future course of action and careful consideration of factors like the assessment of the gestational age, the likelihood of infection, and availability of neonatal intensive care facilities helps to decide the appropriate management ${ }^{1}$. Infection is closely associated either as an etiologic factor or as a consequence of PROM. From the maternal point of view, chorioamnionitis is a major problem that can lead to intrapartum and postpartum sepsis and rarely, septicemia. Fetal deformities as a consequence of longstanding oligohydramnios and neonatal morbidity and mortality due to sepsis and prematurity are the important neonatal problems in PROM $^{1}$. PROM is very often seen in a busy obstetric ward in our country. Proper diagnosis, close monitoring of the patients, appropriate antimicrobial therapy can improve the maternal and fetal outcome.

\section{MATERIALS \& METHODS}


This prospective study was carried out at the Department of Obstetrics \& Gynecology of Coronel Malek Medical College Hospital (CMMCH), Manikganj, Bangladesh from January 2020 to December 2020. From all admitted pregnant patients, only cases of Premature Rupture of Membrane (PROM) were taken for the study.

After formulation of the aim of the study, a clinical data sheet was made for recording all information of the pregnant women. Verbal informed consent was taken from each woman. After history taking, the duration of gestational age was estimated from $1^{\text {st }}$ day of the last menstrual period (LMP), previous antenatal records, clinical examination \& also previous ultrasonography (USG) reports.

Diagnosis of PROM was confirmed from the history of gush of fluid coming from the vagina, demonstration of amniotic fluid leakage from the cervix by a sterile speculum examination $(\mathrm{P} / \mathrm{S})$ or pooling of amniotic fluid in the posterior vaginal fornix, and observation of oligohydramnios by amniotic fluid index (AFI) through USG. During P/S examination, a high vaginal \& endocervical swab was taken from all the patients and sent for culture \& sensitivity, a blood sample for leukocyte count, and urine for routine examination \& culture sensitivity $(\mathrm{C} / \mathrm{S})$ was sent. E.coli was the most common pathogen.

The plan of management of patients with PROM was decided according to the condition of the patient, duration of gestational age, duration of membrane rupture, associated any complicating factors, maternal \& fetal condition, and also neonatal intensive care facility. Prophylactic antibiotic (erythromycin base $250 \mathrm{mg}$ by mouth four times per day for 7 days plus (+) amoxicillin 500mg by mouth three times per day for 7 days) was given to reduce maternal and neonatal infective morbidity and to delay delivery. If the membranes had been ruptured for more than 18 hours, injection ampicillin $2 \mathrm{gm}$ IV every 6 hourly given to help reduce Group B streptococcus infection in the neonate.

Dexamethasone injection $12 \mathrm{mg}$ IM, two doses 12 hours apart was given to the mother to improve fetal lung maturity if the duration of pregnancy was less than 34 weeks.

Conservative management was given when gestational age was less than 37 weeks. But PROM with more than 37 weeks, a vaginal examination $(\mathrm{P} / \mathrm{V})$ was done to assess Bishop's score. After the initial evaluation, $\mathrm{P} / \mathrm{V}$ was restricted to a minimum and the progress of labor was monitored from the nature of uterine contraction \& descent of the fetal head. Delivery was augmented either by medical induction or Lower Uterine Caesarean section (LUCS). C/S was performed when PROM was complicated with other factors.

After delivery, all the babies were assessed by Apgar score and sent to the neonatology dept if any complicating factor was present.

\section{RESULTS}

A total of 297 cases of Premature Rupture of Membrane (PROM) were recorded from January 2020 to December 2020 among 4461 admitted pregnant patients in $\mathrm{CMMCH}$, Manikganj, Bangladesh. Table - I \& II shows that $43 \%$ of the affected women were primigravida and belonged to 20-24 years of age $130(43.7 \%)$, were multigravida $169(56.8 \%)$. One thirty-five $(67.5 \%)$ patients presented with different complications associated with PROM. Table III shows that the most common complication was oligohydramnios (53.3\%) next subclinical urogenital infection $(25.9 \%)$, Pregnancy with medical disease (14.2) \& (5.9\%) women presented with chorioamnionitis. Most of the patients $238(80 \%)$ were delivered by cesarean section $(\mathrm{C} / \mathrm{S})$. Previous $\mathrm{C} / \mathrm{S}$, oligohydramnios, fetal distress, chorioamnionitis were the common indications for doing C/S (Table - III). Only 47 (15.8\%) patients were delivered vaginally, 7 (2\%) patients responded to conservative management, and $5(1.6 \%)$ patients were referred to a tertiary 
center for extreme prematurity with associated complicating factors (previous LUCS with bad obstetrical history). Table-IV shows most of the women delivered by LUCS (80\%). Only 7 women $(2.35 \%)$ could prolong their pregnancy. Table $-\mathrm{V}$ shows that most of the babies, 96 $(32.5 \%)$ had birth weight in between $2.1-2.5 \mathrm{~kg}$, $48(16.1 \%)$ babies had birth weight between 1.6 to $2 \mathrm{~kg}$ and $29(9.7 \%)$ babies were born with less than $1.5 \mathrm{~kg}$ body weight. Immediately after birth, 228(76.7\%) babies were born with Apgar score $>7,58(19.7 \%)$ within 4-6, 10(3.3\%) babies had Apgar score $<3$. Only $1(0.33 \%)$ baby had no sign of life after birth (Table- V). There was no maternal mortality but perinatal death was $7(2.5 \%)$.

Table-I: Age distribution of women of PROM ( $\mathrm{n}=297)$.

\begin{tabular}{|l|c|l|}
\hline Age group & Number of patients & Percentage(\%) \\
\hline $15-19$ & 20 & $6.7 \%$ \\
\hline $20-24$ & 130 & $43.7 \%$ \\
\hline $25-29$ & 87 & $29.5 \%$ \\
\hline $30-34$ & 40 & $13.4 \%$ \\
\hline$>35$ & 20 & $6.7 \%$ \\
\hline
\end{tabular}

In this study, the age of the patients ranged between 15->35 years. Most of the patients belonged to the age group 20-24 years (43.7\%).

Table-II: Gravidity of the women of PROM ( $\mathrm{n}=297)$.

\begin{tabular}{|l|l|l|}
\hline Gravida & $\begin{array}{c}\text { Number of } \\
\text { patients }\end{array}$ & $\begin{array}{c}\text { Percentage } \\
(\mathbf{\%})\end{array}$ \\
\hline 1st & 128 & $43.2 \%$ \\
\hline 2nd & 62 & $20.8 \%$ \\
\hline 3rd & 43 & $14.4 \%$ \\
\hline 4th & 30 & $10.1 \%$ \\
\hline 5th & 20 & $6.7 \%$ \\
\hline 6th & 8 & $2.6 \%$ \\
\hline 7th & 6 & $2.0 \%$ \\
\hline
\end{tabular}

Table II shows that $43.2 \%$ of PROM patients were primigravida and $56.8 \%$ of patients were multigravida.

Table-III: Complications of PROM.

\begin{tabular}{|l|c|c|}
\hline \multicolumn{1}{|c|}{ Indication } & $\begin{array}{c}\text { Number of } \\
\text { patients }\end{array}$ & $\begin{array}{c}\text { Percentage } \\
(\mathbf{\%})\end{array}$ \\
\hline $\begin{array}{l}\text { Pregnancy with subclinical urogenital } \\
\text { infection }\end{array}$ & 35 & $25.9 \%$ \\
\hline Oligohydramnios & 72 & $53.3 \%$ \\
\hline Choioamnionitis & 8 & $5.9 \%$ \\
\hline Pregnancy with Medical disease & 19 & $14.2 \%$ \\
\hline Pregnancy with appendicitis & 1 & $0.74 \%$ \\
\hline
\end{tabular}

Table III shows that the most common complication of was oligohydramnios $(53.3 \%)$ next subclinical urogenital infection (25.9\%), Pregnancy with medical disease $(14.2 \%) \&(5.9 \%)$ women presented with chorioamnionitis.

Table-IV: Delivery outcome $(\mathrm{n}=297)$. 


\begin{tabular}{|l|l|l|}
\hline Type of Delivery & Number of babies & Percentage(\%) \\
\hline LUCS & 238 & 80.2 \\
\hline NVD & 47 & 15.8 \\
\hline Not delivered & 7 & 2.3 \\
\hline Referred & 5 & 1.6 \\
\hline
\end{tabular}

Table IV shows most of the women delivered by LUCS (80.02\%). Only 7 women $(2.3 \%)$ could prolong their pregnancy.

Table-V: Fetal Birth weight ( $\mathrm{n}=297)$.

\begin{tabular}{|l|l|l|}
\hline Fetal weight $(\mathrm{Kg})$ & Number of babies & Percentage (\%) \\
\hline$<1.5$ & 29 & 9.7 \\
\hline $1.6-2$ & 48 & 16.1 \\
\hline $2.1-2.5$ & 96 & 32.5 \\
\hline $2.6-3$ & 80 & 26.9 \\
\hline $3.1-3.5$ & 36 & 12.1 \\
\hline$>3.5$ & 8 & 2.69 \\
\hline
\end{tabular}

Table $\mathrm{V}$ shows most of the babies 96(32.5\%) had birth weight in between $2.1-2.5 \mathrm{~kg}$ and $29(9.7 \%)$ babies were less than $1.5 \mathrm{~kg}$ body weight.

Table-VI: Apgar Score of Babies ( $\mathrm{n}=297)$.

\begin{tabular}{|l|l|l|}
\hline Apgar Score & Number of babies & Percentage (\%) \\
\hline$>7$ & 228 & 76.7 \\
\hline $4-6$ & 58 & 19.7 \\
\hline$<3$ & 10 & 3.36 \\
\hline 0 & 1 & 0.33 \\
\hline
\end{tabular}

The maximum number of babies (76.7\%) had apgar score $>7$ and only 10(3.36\%) babies were born with apgar score $<3$.

\section{DISCUSSION}

To give birth to a healthy baby is a most awaited event in a woman's life. PROM is an important cause of preterm labor and delivery of a low birth weight baby resulting in high perinatal mortality and morbidity ${ }^{3}$. In our country, the true incidence of PROM is very difficult to ascertain because more than $80 \%$ of deliveries occur at home. This study, conducted at $\mathrm{CMMCH}$, shows the incidence of PROM in the hospital was $6.3 \%$. A study undertaken at Dhaka Medical College Hospital (DMCH) in 1995 showed the incidence of PROM to be $8.12 \%^{7}$ and in 2001 it was $9.05 \%$. Another study at Rangpur Medical College Hospital reported an incidence of $9.04 \%{ }^{9}$. The incidence at Indian studies from Mumbai reported by
Bhalerao \& Desai and Bhide in 2000 \& 2001 showed an incidence of PROM between 7 and $12 \%{ }^{10}$. Daftary and Desai 2006 correlated the incidence of PROM with gestational maturity and reported that PROM before the onset of true labor occurred in $5-20 \%$ of all women in labour ${ }^{11}$. Although there is some morbidity when PROM occurs in term pregnancies, the fundamental clinical problem is preterm PROM, a condition that occurs in $3 \%$ of all pregnancies and is responsible for approximately $30 \%$ of all preterm deliveries as reported by Arias and Tomich on $1982^{12}$. During the study period, $57.6 \%$ of patients were admitted at term PROM ( $>37$ weeks) and $42.4 \%$ of patients came prior to 37 weeks (PPROM). In this study, most of the affected patients belong to 20-24 years of age 
$(43.7 \%)$, which is similar to other studies ${ }^{7-9}$. In many studies, high parity is one of the risk factors for increasing PROM $^{7-9}$. In the present study, maximum women were multigravida $(56.8 \%)$ coming from low socio-economic families. Low socioeconomic status is an important risk factor for both PROM and preterm labor. Associated factors such as malnutrition, overexertion, poor hygiene, stress, recurrent genitourinary infections, and anemia considerably increase the risk $^{1}$.In our study, more than $80 \%$ of patients had no ANC. In a study by Begum, $50 \%$ of patients were in the group of low socioeconomic condition having no or irregular antenatal check-up which is almost similar to this study ${ }^{9}$. In 1991, Romero et al reported that infection is twice as frequent in PROM than in preterm labor with intact membranes. In another study by Romero et al in 1993, in term PROM the incidence of infection was approximately $20 \%$ and in PPROM it was $38.3 \%^{13}$. Also, women with PPROM and labor at the time of admission had a greater incidence of chorioamnionitis than women with PPROM admitted without labour ${ }^{13}$. Associated maternal medical and obstetrical complications had a highly significant impact on PROM. During the study period, $77.3 \%$ of PROM patients were admitted with different complications. Among them, $3.1 \%$ of patients presented with chorioamnionitis. About 53\% of patients had maternal complications and $11 \%$ of patients had clinical evidence of chorioamnionitis in the study by Begum ${ }^{9}$. Another study by Miller et al in 1978 and Johnson et al in 1981 observed the prevalence of microbial invasion of the amniotic cavity with PROM as $30 \%{ }^{14}$. In the present study, high vaginal swab culture was positive in $25.8 \%$ of patients. E.coli was the most common pathogen. Because of the limitation of facilities, anaerobic culture and other relevant investigations for diagnosis of subclinical or clinical amniotic fluid infection were not done, only aerobic culture was done. The evidence implicating infection as an etiologic agent in membrane weakening and rupture is robust. Between 14.6 and $38 \%$ of women with PROM will have positive amniotic fluid cultures ${ }^{2}$. The microorganisms isolated from amniotic fluid cultures (Peptostreptococcus, Bacteroides, Fusobacterium, Lactobacillus, Ureaplasma urealyticum) are similar to those normally found in the vagina, strongly suggesting that the source of infection is bacteria normally present in the vagina or the cervix. The mechanism of ascending infection is not clear ${ }^{2}$. Hein et al in 2001 observed that changes in the antibacterial properties of cervical mucus may also play an important role in facilitating ascending infection $^{15}$. The importance of factors that theoretically may increase the possibility of ascending infection such as sexual intercourse, pelvic digital examinations during pregnancy, and history of prior cervical dilatation for abortion or $\mathrm{D} \& \mathrm{C}$ has been disproved by clinical investigations. On the other hand, colonization of the lower genital tract by Chlamydia, Neisseria gonorrhoeae, group B streptococcus, Tricomonas, E.coli, and bacteroid species has been shown to increase the risk of $\mathrm{PROM}^{2}$. In this series, $80.2 \%$ of patients were delivered by cesarean section \& $15.8 .2 \%$ of patients were delivered vaginally. But Begum shows that only $32 \%$ of patients were delivered by $\mathrm{C} / \mathrm{S}^{9}$. Puerperal sepsis $(5.6 \%) \&$ wound infections $(21.25 \%)$ were common puerperal complications that are similar to other studies ${ }^{7-9}$. A reduction in the incidence of puerperal sepsis is due to proper use of antibiotics and timely termination of pregnancy ${ }^{9}$. During our study period, most of the babies $32.5 \%$ born belonged to birth weight between $2.1-2.5 \mathrm{~kg}, 16.1 \%$ of babies were in between 1.6 to $2 \mathrm{~kg}$, and $9.7 \%$ of babies were born with less than $1.5 \mathrm{~kg}$ body weight. Several studies have concluded that hyaline membrane disease is the greatest threat to the newborn when PROM occurs before term. The data from Mercer in 2003 show that at all gestational age the risk of respiratory distress is 
greater than the risk of infection. At 24 weeks $100 \%$ of the newborns develop respiratory distress syndrome, at 32 weeks $25 \%$, and at 34 weeks close to $10 \%$. The risk of sepsis at these gestational ages was 40, 32, 4.5, and $3 \%$, respectively ${ }^{16}$. In this study, all newborn surveillance was not possible because of the limitation of facilities. All newborns with complications like asphyxia, sepsis, low birth weights were sent to the neonatology ward for further management. Perinatal death was $0.33 \%$ in this study but there was no maternal mortality.

\section{CONCLUSION}

Most of the affected women belong to 20-24 years of age $130(43.7 \%)$, about $43 \%$ of them were primigravida and $169(56.8 \%)$ were multigravida. Term PROM was more in comparison to PPROM and most of them were multigravida. The cesarean section rate was high. The most common complication was oligohydramnios $(53.3 \%)$ next subclinical urogenital infection (25.9\%), Pregnancy with medical disease (14.2) \& $(5.9 \%)$ women presented with chorioamnionitis. About $2 / 3$ of the babies birth weight were within 2 to $2.5 \mathrm{~kg}$.

\section{REFERENCES:}

1. Usha R. Krishna and Monisa H.Shah. Prelabour Rupture of Membranes, Obstetrics and Gynecology for Postgraduates, edited by S.S. Ratnam, K. Bhasker Rao and S. Arulkumaran, 2nd edition-1999; Vol-1,96-108.

2. Arias F, Daftary SN, Bhide AG. Premature Rupture of Membrane, Practical Guide to High- Risk Pregnancy \& Delivery, A South Asian Perspective, $3^{\text {rd }}$ edition-2008; 240-261.

3. D.C. Dutta. Prelabour Rupture of the Membranes, Text Book of Obstetrics, $6^{\text {th }}$ edition 2006, 317-320.

4. Pressman EK, Cavanaugh JL, Woods JR, et al. Physical properties of the chrioamnion throughout gestation. Am J Obstetric Gynecology 2002; 187; 672-5.
5. Barabas AP. Ehlers-Danlos syndrome associated with prematurity and premature rupture of fetal membranes; possible increase in incidence. Br Med J 1966; 2: 682-4.

6. McGregor JA. French JL, Lawellin D, et al. Bacterial protease-induced reduction of chorioamnionitis membrane strength and elasticity. Obstet Gynecol 1987; 69: 167-74.

7. Tasnim S: Clinical Profile and Outcome of Pregnancy in Premature Rupture of Membrane -A Study in Dhaka Medical College Hospital. Dissertation; Bangladesh College of Physicians and Surgeons, 1995.

8. Begum A; Maternal and Fetal Outcome of Premature Rupture of Membrane One year Study in Dhaka Medical College Hospital. Dissertation; Bangladesh College of Physicians and Surgeons, 2001.

9. Begum N; Epidemiology of Premature Rupture of Membrane and Management in Rangpur Medical College Hospital. Dissertation; Bangladesh College of Physicians and Surgeons, 2004.

10. Bhalerao S, Desai A. Premature rupture of membranes. Jn: Saraiya UB, Rao KB, Chatterjee A, eds. Principles and Practice of Obstetrics\& Gynecology (2nd edn). An FOGSI Publication. New Delhi: Jaypee Brothers, 2003: 125.

11. Daftary SN, Desai SV. Preterm labor and premature rupture of membranes. In: Daftary SN, Desai SV, eds. Selected Topics in Obstetrics \& Gynecology ( $2^{\text {nd }}$ edn). New Delhi: BI Publications, 2006: 128.

12. Arias F, Tomich PH. Etiology and outcome of low birth weight and preterm infants. Obstetric Gynecology 1982; 60: 277-81.

13. Romero R, Yoon BH, Mazor M, et al. A comparative study of the diagnostic performance of amniotic fluid glucose, 
white cell count, interleukin-6 and Gram stain in the detection of microbial invasion in patients with preterm premature rupture of the membranes. Am J Obstetric Gynecology 1993; 169: 83951.

14. Johnson J WC, Daikoku NH, Niebyl JR et al. Premature rupture of membranes and prolonged latency. Obstetrics Gynecology
1981; 57: 547-556.

15. Hein M, Helmig RB, Schonheyder HC, et al. An in vitro study of antibacterial properties of the cervical mucous plug in pregnancy. Am J Obstetric Gynecology 2001; 185 .

16. Mercer BM. Preterm premature rupture of the membranes. Obstetrics Gynecology 2003; 101: 178-93. 